\title{
HOMOTOPY GROUPS OF SUSPENDED CLASSIFYING SPACES: AN EXPERIMENTAL APPROACH
}

\author{
ANA ROMERO AND JULIO RUBIO
}

\begin{abstract}
When the results of a computer program are compared to some theorems proved on a theoretical basis three situations can occur: there can be an agreement between both approaches, the computer program can obtain calculations not covered by the theorems, or a discrepancy can be found between both methods. In this paper we report on a work where the three above mentioned situations happen. We have enhanced the Computer Algebra called Kenzo to deal with the computation of homotopy groups of suspended classifying spaces, a problem tackled by Mikhailov and $\mathrm{Wu}$ in a paper published in the journal Algebraic and Geometric Topology. Our experimental approach, based on completely different methods from those by Mikhailov and Wu, has allowed us in particular to detect an error in one of their published theorems.
\end{abstract}

\section{Motivation And Statement of the PRoblem}

It is well known that the suspension functor $\Sigma$ applied to a topological space $X$ shifts its homology groups, that is to say, $H_{n}(\Sigma X) \cong H_{n-1}(X)$. However, in the case of homotopy groups the situation is not so favorable and in general there is not a direct relation between $\pi_{*}(\Sigma X)$ and $\pi_{*}(X)$. Let us consider for instance a circle $S^{1}$, whose homotopy type is very simple. The homology groups of the suspension $\Sigma S^{1}=S^{2}$ are obvious but the problem of computing the homotopy groups of $S^{2}$ is a difficult problem in Algebraic Topology.

Given a group $G$, its classifying space, that is, the Eilenberg-MacLane space $K(G, 1)$ [10], has trivial homotopy groups: $\pi_{1}(K(G, 1)) \cong G$ and $\pi_{n}(K(G, 1))=0$ for each $n \neq 1$. But when applying the suspension functor $\Sigma$, the new homotopy groups $\pi_{*}(\Sigma K(G, 1))$ are in general unknown. In [11], several groups $\pi_{n}(\Sigma K(G, 1))$ are obtained for some particular cases of $G$ and $n$, making use of different results and techniques from group theory and homotopy theory. More concretely, the main results of the article by Mikhailov and $\mathrm{Wu}$ are descriptions of the groups $\pi_{4}(\Sigma K(A, 1))$ and $\pi_{5}(\Sigma K(A, 1))$ when $A$ is any finitely generated Abelian group; as applications, they also determine $\pi_{n}(\Sigma K(G, 1))$ with $n=4$ and 5 for some nonAbelian groups, as $G=\Sigma_{3}$ the third symmetric group and $G=S L(\mathbb{Z})$ the standard linear group, and $\pi_{4}\left(\Sigma K\left(A_{4}, 1\right)\right)$ for $A_{4}$ the fourth alternating group.

The goal of this work consists in trying to obtain a computer program calculating $\pi_{*}(\Sigma K(G, 1))$ for a general group $G$, which will allow one to obtain as particular computations some theoretical results of [11. To this aim, we have developed some algorithms implemented in the Computer Algebra system Kenzo [4. Thanks

\footnotetext{
Received by the editor October 7, 2011 and, in revised form, January 11, 2012.

2010 Mathematics Subject Classification. Primary 68W05, 55-04, 55Q99.

Both authors were partially supported by Ministerio de Ciencia e Innovación, Spain, project MTM2009-13842-C02-01.
} 
to our programs, groups $\pi_{4}(\Sigma K(A, 1))$ and $\pi_{5}(\Sigma K(A, 1))$ have been computed for several finitely generated Abelian groups $A$, reproducing the same results obtained in [11. We have also experimentally determined some new groups $\pi_{6}(\Sigma K(A, 1))$ (which do not appear in [1] ) and $\pi_{n}(\Sigma K(G, 1)$ ) for other non-Abelian groups $G$ not considered in that article. Moreover, our experiments have made it possible to detect an error in Mikhailov and Wu's paper. The authors state in Theorem 5.4:

Let $A_{4}$ be the fourth alternating group. Then $\pi_{4}\left(\Sigma K\left(A_{4}, 1\right)\right)=\mathbb{Z}_{4} 1$

Our programs produce a different result, namely $\pi_{4}\left(\Sigma K\left(A_{4}, 1\right)\right)=\mathbb{Z}_{12}$. The authors of the paper inadvertently forgot the 3-primary component, as they have admitted in a private communication.

The paper is organized as follows. After this brief introduction to our problem, in Section 2 we explain the method we have followed in order to determine in a computational way the homotopy groups of suspended classifying spaces. This method is based on the effective homology technique and is implemented in the Kenzo system. Section 3 presents some examples of our computations and a table with our final results. The paper ends with a section of conclusions and further work.

\section{The Method: Effective homology And the KenZo System}

The Kenzo system 4, developed by Francis Sergeraert and some co-workers, is a Common Lisp program devoted to Symbolic Computation in Algebraic Topology. In particular, it makes it possible to determine homology and homotopy groups of complicated spaces and has obtained some results (for example homology groups of iterated loop spaces of a loop space modified by a cell attachment, components of complex Postnikov towers, etc.) which were not known before. One of the important characteristics of Kenzo is that it can deal with topological spaces of infinite nature, which are encoded using functional programming; homology and homotopy groups of these spaces are then computed by means of the effective homology method, introduced in [18] and deeply explained in [15].

The effective homology method is based on the following idea: on the one hand, if a topological space $X$ has finite nature, then its homology groups can be determined by means of an elementary algorithm based on some matrix operations. On the other hand, if $X$ has infinite dimension, then Kenzo should be able to find a homotopy equivalence between a chain complex canonically associated with $X$, $C_{*}(X)$, and an effective (finite type) chain complex $E_{*}$; this equivalence is denoted $C_{*}(X) \Longleftrightarrow E_{*}$ and we say then that $X$ has effective homology. This particular homotopy equivalence (which must satisfy some special properties, see [15] for the formal definition) makes it possible to determine the homology groups of $X$ by means of those of $E_{*}$ in an explicit way: the equivalence provides not only a formal description of the groups but also their generators, which are important in order to make calculations inside the groups or use them in other constructions.

The difficult part of the process consists in obtaining the effective chain complex $E_{*}$ and the desired equivalence $C_{*}(X) \Longleftrightarrow E_{*}$ for a given topological space $X$. This is done thanks to the following general idea: given some topological spaces $X_{1}, \ldots, X_{n}$, a topological constructor $\Phi$ produces a new topological space $X$. If effective homology versions of the spaces $X_{1}, \ldots, X_{n}$ are known (that is to say, one

\footnotetext{
${ }^{1}$ In this paper an expression as $\mathbb{Z}_{4}$ will denote, as usual in Algebraic Topology, the finite Abelian group $\mathbb{Z} / 4 \mathbb{Z}$; the same group is denoted in Mikhailov and Wu's paper as $\mathbb{Z} / 4$.
} 


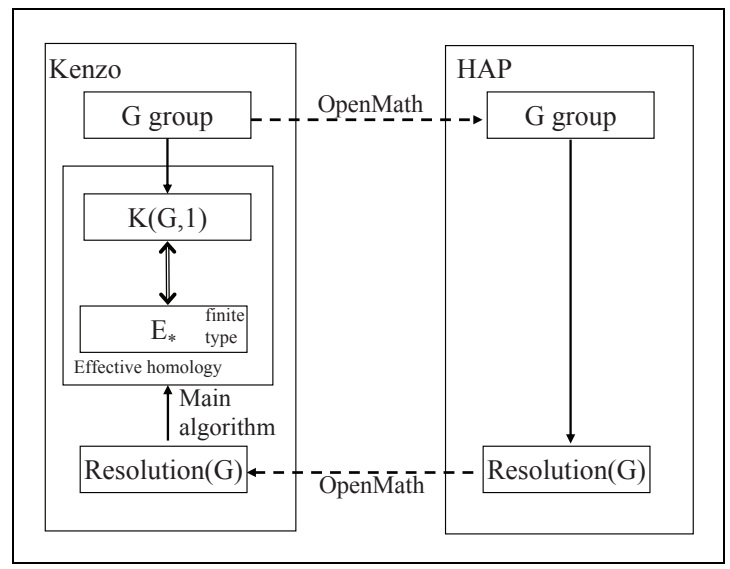

Figure 1. Communication between Kenzo and HAP

has equivalences $C_{*}\left(X_{i}\right) \Longleftrightarrow E_{i}$ for each $i$ where $E_{i}$ is a finite type chain complex), then one should be able to build an effective homology version of the space $X$, and this version would allow us to compute the homology groups of $X$.

Given a group $G$, its classifying space is an Eilenberg-MacLane space $K(G, 1)$, which is a Kan simplicial set satisfying $\pi_{1}(K(G, 1)) \cong G$ and $\pi_{n}(K(G, 1))=0$ for $n \neq 1$, and its homology groups are by definition the homology groups of $G$. From the definition of simplicial classifying space in 10, one can observe that the standard Eilenberg-MacLane space satisfies $K(G, 1)_{n}=G^{n}$ for $n>1$. In particular, if $G=\mathbb{Z}$ (or any group with an infinite number of elements), then the space $K(G, 1)$ is infinite and one cannot directly compute its homology groups. If the group $G$ is finite, then $K(G, 1)$ is finitely generated in each dimension, but the number of generators is very large and in most cases complexity problems prevent the actual computation of $H_{*}(K(G, 1))$, even in low degrees.

Eilenberg-MacLane spaces have been implemented in Kenzo (using functional programming), and can be constructed by means of a function which inputs a group $G$ and returns a simplicial model of the space $K(G, 1)$. In order to compute its homology groups, we have developed an algorithm determining the effective homology of $K(G, 1)$ from a resolution [2] of the group $G$, supposing that the resolution has finite dimension in each degree. This algorithm is completely described in [12]. For some groups (for instance, cyclic groups $\mathbb{Z}_{n}$ ), the needed resolution is directly implemented in the Kenzo system; in other cases, the resolution is obtained from the GAP Computer Algebra program [17] thanks to the HAP package [5], which was developed by Graham Ellis and is devoted to computing homology of groups. After determining the finite resolution by using HAP, it is translated into Kenzo by means of an OpenMath processor, as described in Figure 1. Once the resolution is installed in Kenzo, our algorithm is applied to compute the effective homology of $K(G, 1)$, that is, an equivalence between $C_{*}(K(G, 1))$ and an effective chain complex $E_{*}$. See [12] for details on the complete process.

Once we have the space $K(G, 1)$ in Kenzo with its effective homology, one can determine its homology groups as explained before. Moreover, we can use this space inside other constructions in Algebraic Topology producing new spaces with 
effective homology, allowing the computation for example of homology groups of 2types and central extensions, as presented in 13 . One can also apply the suspension functor, obtaining a new simplicial set $\Sigma K(G, 1)$; since an effective homology version of $\Sigma$ is implemented in Kenzo, the suspended classifying space $\Sigma K(G, 1)$ is again an object with effective homology. Therefore, if $G$ is a group and we have a finite resolution for it (directly constructed or obtained by means of HAP), the homology groups of $\Sigma K(G, 1)$ can be determined.

Although the process is not so direct as for homology groups, Kenzo is also able to determine the homotopy groups of a simplicial set with effective homology, and in particular it can compute the desired $\pi_{*}(\Sigma K(G, 1)$ ) (whenever we have a finite resolution of the group $G$ ). The technique used by Kenzo for computing homotopy groups is based on the Whitehead tower method, and is described in the documentation of the program 4 .

In the following section we present some examples of calculations of homotopy groups of suspended classifying spaces $\Sigma K(G, 1)$ and a table with our final results.

\section{Computations and Results}

Let us consider for example $G=\mathbb{Z}_{5}$, the cyclic group with 5 elements. It is built in Kenzo with the following instruction:

$>$ ( $\operatorname{setf}$ z5 (cyclicgroup 5))

[K1 Abelian-Group]

The result is an Abelian group, stored in the variable z5. The Eilenberg-MacLane space $K\left(\mathbb{Z}_{5}, 1\right)$ is constructed by means of the function $\mathrm{k}-\mathrm{g}-1$, and is an Abelian simplicial group:

$>$ ( $\operatorname{setf} \mathrm{k}-\mathrm{z} 5-1(\mathrm{k}-\mathrm{g}-1 \mathrm{z} 5))$

[K2 Abelian-Simplicial-Group]

The effective homology of $K\left(\mathbb{Z}_{5}, 1\right)$ is directly built by our programs making use of a well-known finite resolution of the group $\mathbb{Z}_{5}$ (see 2 for details), that we have implemented in Kenzo. The effective homology of an object is stored in the slot efhm and can be obtained as follows:

$>$ (efhm k-z5-1)

[K50 Homotopy-Equivalence $\mathrm{K} 2<=\mathrm{K} 40 \Rightarrow \mathrm{K} 31$ ]

It is an equivalence between $\mathrm{K} 2$, that is, the space $K\left(\mathbb{Z}_{5}, 1\right)$, and an effective chain complex $K 31$; the effective homology of $K\left(\mathbb{Z}_{5}, 1\right)$ makes it possible to determine its homology groups:

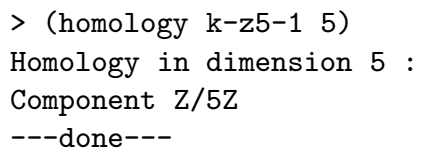

This is the well-known result [2] $H_{5}\left(K\left(\mathbb{Z}_{5}, 1\right)\right) \equiv H_{5}\left(\mathbb{Z}_{5}\right)=\mathbb{Z}_{5}$.

We now apply the suspension functor $\Sigma$ to build the suspended classifying space $\Sigma K\left(\mathbb{Z}_{5}, 1\right)$ :

$>$ ( $\operatorname{setf} \mathrm{s}-\mathrm{k}-\mathrm{z} 5-1$ (suspension $\mathrm{k}-\mathrm{z} 5-1)$ )

[K51 Simplicial-Set]

This is again an object with effective homology:

$>$ (efhm $\mathrm{s}-\mathrm{k}-\mathrm{z} 5-1)$

[K68 Homotopy-Equivalence K51 <= K56 $\Rightarrow$ K62] 
The system Kenzo itself does not include a function for computing homotopy groups as it does for homology, but it makes it possible to compute homotopy groups following the Whitehead tower method as it is explained in the documentation of the program. Jónathan Heras has automated this construction by means of a new function homotopy, which was written as part of the friendly interface for Kenzo called fKenzo [9]. The function homotopy gives us the desired homotopy groups of $\left.\Sigma K\left(\mathbb{Z}_{5}, 1\right)\right)$, as presented in the following lines:

$>$ (homotopy $\mathrm{s}-\mathrm{k}-\mathrm{z} 5-1$ 5)

Homotopy in dimension 5 :

Component $\mathrm{Z} / 5 \mathrm{Z}$

Component $\mathrm{Z} / 5 \mathrm{Z}$

---done---

In this way we obtain the group $\pi_{5}\left(\Sigma K\left(\mathbb{Z}_{5}, 1\right)\right)=\mathbb{Z}_{5} \oplus \mathbb{Z}_{5}$, which corresponds to the result given in 11 .

The process is similar for other cyclic groups $\mathbb{Z}_{n}, n \geq 2$, and for $\mathbb{Z}$ the infinite cyclic group (an easy finite resolution is known for these groups (see [2]) and has been implemented in Kenzo, so that one can directly determine the effective homology of $K(\mathbb{Z}, 1)$ and $\left.K\left(\mathbb{Z}_{n}, 1\right)\right)$. One can also compute the homotopy groups of $\Sigma K(A, 1))$ where $A$ is a finitely generated Abelian group. Thanks to the well-known isomorphism $K\left(A_{1} \oplus A_{2}, 1\right) \cong K\left(A_{1}, 1\right) \times K\left(A_{2}, 1\right)$, the desired space $K(A, 1)$ is constructed as a Cartesian product of different factors $K\left(\mathbb{Z}_{n}, 1\right)$ and $K(\mathbb{Z}, 1)$ by means of the Kenzo function called crts-prdc, which automatically determines the effective homology of the desired space. Let us consider for example the case $A=\mathbb{Z}_{3} \oplus \mathbb{Z}_{3}$; the following lines include the Kenzo instructions for computing $\pi_{4}\left(\Sigma K\left(\mathbb{Z}_{3} \oplus \mathbb{Z}_{3}, 1\right)\right)=\mathbb{Z}_{3} \oplus \mathbb{Z}_{3} \oplus \mathbb{Z}_{3} \oplus \mathbb{Z}_{3} \oplus \mathbb{Z}_{3} \oplus \mathbb{Z}_{3} \oplus \mathbb{Z}_{3} \oplus \mathbb{Z}_{3} \equiv \mathbb{Z}_{3}^{\oplus 8}:$

$>$ ( $\operatorname{setf}$ z3 (cyclicgroup 3))

[K627 Abelian-Group]

$>($ setf $\mathrm{k}-\mathrm{z} 3-1 \quad(\mathrm{k}-\mathrm{g}-1 \mathrm{z} 3))$

[K628 Abelian-Simplicial-Group]

$>$ ( $\operatorname{setf} \mathrm{k}-\mathrm{z} 3+3-1$ (crts-prdc k-z3-1 k-z3-1))

[K633 Simplicial-Set]

$>($ setf $\mathrm{s}-\mathrm{k}-\mathrm{z} 3+3-1$ (suspension $\mathrm{k}-\mathrm{z} 3+3-1))$

[K640 Simplicial-Set]

$>$ (homotopy $\mathrm{s}-\mathrm{k}-\mathrm{z} 3+3-14$ )

Homotopy in dimension 4 :

Component $\mathrm{Z} / 3 \mathrm{Z}$

Component $\mathrm{Z} / 3 \mathrm{Z}$

Component $\mathrm{Z} / 3 \mathrm{Z}$

Component $\mathrm{Z} / 3 \mathrm{Z}$

Component $\mathrm{Z} / 3 \mathrm{Z}$

Component Z/3Z

Component $\mathrm{Z} / 3 \mathrm{Z}$

Component $\mathrm{Z} / 3 \mathrm{Z}$

---done---

For the case of symmetric groups $\Sigma_{3}$ and $\Sigma_{4}$ and the alternating group $A_{4}$, the corresponding finite resolutions are not so simple and we have not implemented them in Kenzo. We obtain them thanks to the HAP package [5] of the GAP Computer Algebra system [17] and import them into Kenzo by means of a specific OpenMath processor. See Figure 1 and 12] for the details of this communication. 
TABLE 1

\begin{tabular}{|r|c|c|c|c|c|}
\hline & $\pi_{2}$ & $\pi_{3}$ & $\pi_{4}$ & $\pi_{5}$ & $\pi_{6}$ \\
\hline \hline$\Sigma K(\mathbb{Z}, 1) \simeq S^{2}$ & $\mathbb{Z}$ & $\mathbb{Z}$ & $\mathbb{Z}_{2}$ & $\mathbb{Z}_{2}$ & $\mathbb{Z}_{12}$ \\
\hline$\Sigma K\left(\mathbb{Z}_{2}, 1\right)$ & $\mathbb{Z}_{2}$ & $\mathbb{Z}_{2}$ & $\mathbb{Z}_{4}$ & $\mathbb{Z}_{2}{ }^{\oplus 2}$ & $\mathbb{Z}_{2}{ }^{\oplus 2}$ \\
\hline$\Sigma K\left(\mathbb{Z}_{3}, 1\right)$ & $\mathbb{Z}_{3}$ & $\mathbb{Z}_{3}$ & $\mathbb{Z}_{3}$ & $\mathbb{Z}_{3}{ }^{\oplus 2}$ & $\mathbb{Z}_{3}{ }^{\oplus 5}$ \\
\hline$\Sigma K\left(\mathbb{Z}_{4}, 1\right)$ & $\mathbb{Z}_{4}$ & $\mathbb{Z}_{4}$ & $\mathbb{Z}_{2} \oplus \mathbb{Z}_{4}$ & $\mathbb{Z}_{2} \oplus \mathbb{Z}_{4}{ }^{\oplus 2}$ & \\
\hline$\Sigma K\left(\mathbb{Z}_{2} \oplus \mathbb{Z}_{2}, 1\right)$ & $\mathbb{Z}_{2}{ }^{\oplus 2}$ & $\mathbb{Z}_{2}{ }^{\oplus 4}$ & $\mathbb{Z}_{2}{ }^{\oplus 4} \oplus \mathbb{Z}_{4}{ }^{\oplus 4}$ & & \\
\hline$\Sigma K\left(\mathbb{Z}_{5}, 1\right)$ & $\mathbb{Z}_{5}$ & $\mathbb{Z}_{5}$ & $\mathbb{Z}_{5}$ & $\mathbb{Z}_{5}{ }^{\oplus 2}$ & \\
\hline$\Sigma K\left(\mathbb{Z}_{6}, 1\right)$ & $\mathbb{Z}_{6}$ & $\mathbb{Z}_{6}$ & $\mathbb{Z}_{12}$ & $\mathbb{Z}_{6}{ }^{\oplus 2}$ & \\
\hline$\Sigma K\left(\mathbb{Z}_{7}, 1\right)$ & $\mathbb{Z}_{7}$ & $\mathbb{Z}_{7}$ & $\mathbb{Z}_{7}$ & $\mathbb{Z}_{7}{ }^{\oplus 2}$ & \\
\hline$\Sigma K\left(\mathbb{Z}_{8}, 1\right)$ & $\mathbb{Z}_{8}$ & $\mathbb{Z}_{8}$ & $\mathbb{Z}_{2} \oplus \mathbb{Z}_{8}$ & $\mathbb{Z}_{2} \oplus \mathbb{Z}_{8}{ }^{\oplus 2}$ & \\
\hline$\Sigma K\left(\mathbb{Z}_{9}, 1\right)$ & $\mathbb{Z}_{9}$ & $\mathbb{Z}_{9}$ & $\mathbb{Z}_{9}$ & $\mathbb{Z}_{9}{ }^{\oplus 2}$ & \\
\hline$\Sigma K\left(\mathbb{Z}_{3} \oplus \mathbb{Z}_{3}, 1\right)$ & $\mathbb{Z}_{3}{ }^{\oplus 2}$ & $\mathbb{Z}_{3}{ }^{\oplus 4}$ & $\mathbb{Z}_{3}{ }^{\oplus 8}$ & & \\
\hline$\Sigma K\left(\Sigma_{3}, 1\right)$ & $\mathbb{Z}_{2}$ & $\mathbb{Z}_{2}$ & $\mathbb{Z}_{12}$ & $\mathbb{Z}_{2}{ }^{\oplus 2}$ & \\
\hline$\Sigma K\left(\Sigma_{4}, 1\right)$ & $\mathbb{Z}_{2}$ & $\mathbb{Z}_{2}{ }^{\oplus 2}$ & $\mathbb{Z}_{2} \oplus \mathbb{Z}_{4} \oplus \mathbb{Z}_{8} \oplus \mathbb{Z}_{3}$ & & \\
\hline$\Sigma K\left(A_{4}, 1\right)$ & $\mathbb{Z}_{3}$ & $\mathbb{Z}_{6}$ & $\mathbb{Z}_{12}$ & & \\
\hline
\end{tabular}

Once the resolutions are installed in Kenzo, the process for computing the homotopy groups of the desired $\Sigma K(G, 1))$ is exactly the same as the construction of $\pi_{5}\left(\Sigma K\left(\mathbb{Z}_{5}, 1\right)\right)$.

The Kenzo program can be downloaded and installed from [4, and works under different Lisp environments. Our new programs computing the effective homology of spaces $K(G, 1)$ (and also the GAP code producing the OpenMath representation of a resolution) can be found in www. unirioja.es/cu/anromero/research2.html. Jónathan Heras' homotopy function can be reached in the "Other documents" link in Heras' web page at http://www. unirioja.es/cu/joheras. Finally, in http:// www .unirioja.es/cu/anromero/suspended-classifying-spaces-examples.cl we have included a file with several examples of calculations.

Some results obtained by our programs are shown in Table 1. Let us emphasize that the groups $\pi_{4}(\Sigma K(G, 1))$ and $\pi_{5}(\Sigma K(G, 1)$ in the first twelve rows are the same as those obtained in [11. The results $\pi_{6}\left(\Sigma K\left(\mathbb{Z}_{2}, 1\right)\right)=\mathbb{Z}_{2}^{\oplus 2}=\mathbb{Z}_{2} \oplus \mathbb{Z}_{2}$ and $\pi_{6}\left(\Sigma K\left(\mathbb{Z}_{3}, 1\right)\right)=\mathbb{Z}_{3}^{\oplus 5}=\mathbb{Z}_{3} \oplus \mathbb{Z}_{3} \oplus \mathbb{Z}_{3} \oplus \mathbb{Z}_{3} \oplus \mathbb{Z}_{3}$ are new; they do not appear in the previous article and, to our knowledge, in any other reference. The symmetric group $\Sigma_{4}$ was not considered in [11], so that the homotopy groups of $\Sigma K\left(\Sigma_{4}, 1\right)$ were not known. Finally, let us remark that the result $\pi_{4}\left(\Sigma K\left(A_{4}, 1\right)\right)=\mathbb{Z}_{12}$ does not coincide with the group determined by Mikhailov and Wu: they assert in Theorem 5.4 that $\pi_{4}\left(\Sigma K\left(A_{4}, 1\right)\right)=\mathbb{Z}_{4}$, but our experimental approach has made it possible to detect an error in their proof: as they have admitted, they had forgotten the third primary component.

\section{Conclusions And Further WORK}

In this paper we have illustrated how a computer-based experimental approach can be useful even in sophisticated mathematical domains, as advanced Algebraic Topology. In particular we have computed, by using the Kenzo system, some homotopy groups of suspended classifying spaces. Since most of our results coincide with those presented in [11, it can be considered a good testing site (for both our 
programs and the theoretical results). Nevertheless, when a discrepancy occurs, as in the computation of $\pi_{4}\left(\Sigma K\left(A_{4}, 1\right)\right)$, a challenge is presented to the software engineer: are the programs correct? Even if in this case it was easily checked that the bug was in the theoretical side (in fact, it can be considered an oversight in the transcription of the group rather than an actual error), the problem of the reliability of the software is a difficult one. Let us stress that even if the algorithms to compute homotopy groups by means of the effective homology method have been proved correct, in a traditional way, in several papers (as [18, for example), this is not the same for the programs implementing those algorithms. To increase the reliability of our experimental results, formal methods are being used for the analysis of our algorithms and programs (see, for instance, some works which use respectively the proving assistants Isabelle and Coq in [1] and [3]).

With respect to future improvements of our tools two big lines are open: efficiency and scope. The results presented in Table 1 show the computational limits of our programs. For instance, the computation of $\pi_{6}\left(\Sigma K\left(\mathbb{Z}_{3}, 1\right)\right)$ needed 28 hours of CPU time on an Intel Xeon E5420 computing server with 2.50GHz. Other groups (as the symmetric group $\Sigma_{3}$ ) needed too many resources, exhausting the memoir capabilities of the server (with $8 \mathrm{~Gb}$ of RAM). Let us point out that several algorithms of exponential nature are present in our calculations, and therefore one cannot expect a quick computation of homotopy groups. But, on the other way around, that means that many improvements are possible in the implementation techniques to gain, let us say, one or two dimensions in a theoretically important group. One of the most hopeful approaches is that based on Discrete Morse Theory [7] which could be systematically used in effective homology (see some first contributions in [14]).

As for the scope of our programs, the most outstanding case covered by [11] and not by our methods, is that of the special linear group $S L(\mathbb{Z})$; since we (and HAP) do not know a finite resolution for this group, we cannot apply our algorithms computing the homotopy groups of $\Sigma K(S L(\mathbb{Z}), 1)$. It is in fact a difficult and well-known problem which connects the homotopy groups of suspended classifying spaces with the determination of K-theory groups (for instance, Mikhailov and Wu claim that $\pi_{3}(\Sigma K(S L(\mathbb{Z}), 1)) \cong K_{2}(\mathbb{Z})$ and $\pi_{4}(\Sigma K(S L(\mathbb{Z}), 1)) \cong K_{3}(\mathbb{Z})$. Our idea is to approximate the effective homology of $S L(\mathbb{Z})$ by means of that of the groups $S L_{n}(\mathbb{Z})$. Up to now, Jónathan Heras has been capable of determining, using Kenzo, the first homotopy groups of $\Sigma K\left(S L_{2}(\mathbb{Z}), 1\right)[8$. We are now working in the determination of a finite resolution for $S L_{3}(\mathbb{Z})$, necessary to construct the effective homology of $K\left(S L_{3}(\mathbb{Z}), 1\right)$. To this aim, we are trying to follow the method explained in [6] and the classical approach to the cohomology of $S L_{3}(\mathbb{Z})$ presented in [19] (and more concretely the detailed presentation given in [16]).

\section{ACKNOWLEDGEMENTS}

Thanks are due to Graham Ellis, who told us about the results of [11] and proposed that we try to compute homotopy groups of suspended classifying spaces by means of Kenzo.

\section{REFERENCES}

1. J. Aransay, C. Ballarin, and J. Rubio, A mechanized proof of the basic perturbation lemma, Journal of Automated Reasoning 40 (2008), no. 4, 271-292. MR2418280(2009d:03024) 
2. K. S. Brown, Cohomology of groups, Springer-Verlag, 1982. MR672956 (83k:20002)

3. C. Domínguez and J. Rubio, Effective homology of bicomplexes, formalized in Coq, Theoretical Computer Science 412 (2011), no. 11, 962-970. MR2796695

4. X. Dousson, J. Rubio, F. Sergeraert, and Y. Siret, The Kenzo program, Institut Fourier, Grenoble, 1999, http://www-fourier.ujf-grenoble.fr/ sergerar/Kenzo/.

5. G. Ellis, HAP - Homological Algebra Programming, a package for the GAP system, 2006, http://www.gap-system.org/Packages/hap.html.

6. G. Ellis, J. Harris, and E. Sköldberg, Polytopal resolutions for finite groups, J. Reine Angew. Math 598 (2006), 131-137. MR2270569 (2008g:20117)

7. R. Forman, Morse theory for cell complexes, Advances in Mathematics 134 (1998), 90-145. MR.1612391 (99b:57050)

8. J. Heras, Mathematical knowledge management in algebraic topology, Ph.D. thesis, Universidad de La Rioja, 2011, http://www.unirioja.es/cu/joheras/Thesis/.

9. J. Heras, V. Pascual, J. Rubio, and F. Sergeraert, fKenzo: a user interface for computations in Algebraic Topology, Journal of Symbolic Computation 46 (2011), no. 6, 685-698. MR2781947 (2012a:55002)

10. J. P. May, Simplicial objects in Algebraic Topology, Van Nostrand Mathematical Studies, University of Chicago Press, 1967. MR0222892 (36:5942)

11. R. Mikhailov and J. Wu, On homotopy groups of the suspended classifying spaces, Algebraic and Geometric Topology 10 (2010), 565-625. MR2602844(2011d:55016)

12. A. Romero, G. Ellis, and J. Rubio, Interoperating between computer algebra systems: computing homology of groups with Kenzo and GAP, International Symposium on Symbolic and Algebraic Computation, ISSAC 2009, ACM, 2009, pp. 303-310. MR2742718

13. A. Romero and J. Rubio, Computing the homology of groups: the geometric way, Preprint. http://arxiv.org/abs/1107.3396, 2011.

14. A. Romero and F. Sergeraert, Discrete Vector Fields and fundamental Algebraic Topology, Preprint. http://arxiv.org/abs/1005.5685v1, 2010.

15. J. Rubio and F. Sergeraert, Constructive Homological Algebra and Applications, Lecture Notes Summer School on Mathematics, Algorithms, and Proofs, University of Genova, 2006, http:// www-fourier.ujf-grenoble.fr/ sergerar/Papers/Genova-Lecture-Notes.pdf.

16. R. Sánchez-García, Equivariant K-homology of the classifying space for proper actions, Ph.D. thesis, University of Southampton, 2005, http://www.math.uni-duesseldorf.de/ sanchez/ thesis.pdf. MR2715961

17. M. Schönert et al., GAP - Groups, Algorithms, and Programming, Version 4.4.12, 2008, http://www.gap-system.org.

18. F. Sergeraert, The computability problem in Algebraic Topology, Advances in Mathematics 104 (1994), no. 1, 1-29. MR1272067 (95c:55017)

19. C. Soulé, The cohomology of $S L_{3}(Z)$, Topology 17 (1978), 1-22. MR0470141(57:9908)

Departamento de Matemáticas y Computación, Universidad de La Rioja, Edificio Vives, C/ Luis de UlloA S/N, 26004 Logroño, Spain

E-mail address: ana.romero@unirioja.es

Departamento de Matemáticas y Computación, Universidad de La Rioja, Edificio Vives, C/ Luis de UlloA S/n, 26004 Logroño, Spain

E-mail address: julio.rubio@unirioja.es 